\title{
Conocimientos, actitudes y prácticas en salud bucal de padres y cuidadores en hogares infantiles, Colombia
}

\author{
Farith González Martínez, MSP,(I) Carmen Cecilia Sierra Barrios, Odontol,(I) \\ Luz Edilma Morales Salinas, Odontol.(I)
}

\begin{abstract}
González-Martínez F, Sierra-Barrios CC, Morales-Salinas LE. Conocimientos, actitudes y prácticas en salud bucal de padres y cuidadores en hogares infantiles, Colombia Salud. Publica Mex 20 I ; 53:247-257.
\end{abstract}

\begin{abstract}
Resumen
Objetivo. Describir conocimientos, actitudes y prácticas en salud oral de padres y cuidadores. Material y métodos. Un total de 333 padres y ocho cuidadores de hogares infantiles de Colombia, durante 2010, diligenciaron encuestas y entrevistas de grupos focales. Los datos se analizaron por frecuencia, usando la prueba $\chi^{2}$ para significancia. La información cualitativa se interpretó usando comentarios triangulados, buscando patrones y discrepancias. Resultados. En los padres se observaron buenos niveles de conocimientos $(58,9 \%)$ y actitudes favorables $(74,5 \%)$. Para las prácticas, $50,6 \%$ de los niños duermen con los dientes cepillados y $69,6 \%$ de los padres colocan el dentífrico sobre el cepillo. En los cuidadores, se perciben actitudes positivas para desarrollar estrategias promocionales, pero consideran que los padres son los principales responsables de implementar hábitos orales saludables. Conclusiones. Los padres y cuidadores manifiestan condiciones favorables dentro de sus percepciones, lo que puede ser una oportunidad para promocionar hábitos higiénicos en los niños.
\end{abstract}

Palabras clave: conocimientos; actitudes; prácticas; caries de la infancia temprana; padres; cuidadores; Colombia.
González-Martínez F,

Sierra-Barrios CC, Morales-Salinas LE.

Knowledge, attitudes and practices

in oral health of parents and caregivers

in children's homes in Colombia.

Salud Publica Mex 20II; 53:247-257.

\section{Abstract}

Objectives. To describe knowledge, attitudes and practices in oral health of parents and caregivers. Material and Methods. A total of 333 parents and eight caregivers in children's homes in Colombia in 2010 completed questionnaires and participated in focus group interviews. The data was analyzed for frequency using the $\chi^{2}$ test to evaluate significance. The qualitative information was interpreted using triangulated comments to detect patterns and discrepancies. Results. For parents, good levels of knowledge $(58.9 \%)$ and favorable attitudes $(74.5 \%)$ were observed. In terms of practices, $50.6 \%$ of the children brushed their teeth before bed, with $69.6 \%$ of the parents applying the toothpaste to the brush. Among caregivers, a positive attitude toward developing promotional strategies was perceived, but they considered parents to have the main responsibility in matters of healthy oral habits. Conclusions. Parents and caregivers demonstrated favorable conditions in terms of their perceptions, which can be considered an opportunity to promote hygiene habits in children.

Key Words: knowledge; attitudes; practices; oral health; parents, caregivers; Colombia.

(I) Facultad de Odontología, Universidad de Cartagena, Cartagena, Colombia

Fecha de recibido: 22 de septiembre de 2010 - Fecha de aceptado: 7 de junio de 2011 Solicitud de sobretiros: Farith González Martínez. Facultad de Odontología, Universidad de Cartagena, Colombia. Campus de la salud. Barrio Zaragocilla, Cartagena de Indias.

Correo electrónico: farithgm@hotmail.com. 
$\mathrm{E}_{\mathrm{i}}^{\mathrm{n}}$ las diferentes regiones de un país se considera importante conocer las necesidades en salud de los individuos antes de realizar cualquier tipo de intervención, orientándose inicialmente por los niveles de conocimientos que se tienen sobre la etiología y prevención de estas alteraciones, así como las prácticas diarias de higiene y la actitud frente a la misma. Además, al indagar en niños menores de cinco años, cualquier solicitud debe ir dirigida al personal encargado de su cuidado, tal como lo recomiendan las Normas Técnicas de Atención Preventiva en Salud Bucal del Sistema General de Seguridad social en Salud. ${ }^{1}$

Anivel epidemiológico, varias poblaciones del caribe colombiano se consideran de alto riesgo para las enfermedades bucales, debido al poco acceso que tienen a los servicios de salud y a prácticas de higiene bucal inadecuadas, probablemente influidas por el contexto sociocultural en el cual están inmersos. En la ciudad de Cartagena se han realizado varios estudios que confirman la alta frecuencia de caries dental en niños menores de cinco años. En hogares infantiles, González y colaboradores ${ }^{2}$ encontró prevalencias de $55 \%$ y Díaz y colaboradores ${ }^{3}$ informó de $51 \%$ en preescolares. Ambos estudios utilizaron los criterios diagnósticos visuales de la International Caries Detection and Assessment System (ICDAS). ${ }^{4}$

Para comprender la distribución de las alteraciones bucales dentro de una población son útiles las encuestas de conocimientos, actitudes y prácticas (CAP). En Washington, EUA, Watson y colaboradores ${ }^{5}$ informó que $7 \%$ de los padres de niños en estas edades conocían el propósito de los sellantes y $52 \%$ el del flúor; el motivo más frecuente de búsqueda de asistencia odontológica fue por dolor o urgencia dental. Del mismo modo, en Detroit, Michigan, Finlayson y colaboradores ${ }^{6}$ informaron que las madres tienen una mayor influencia sobre el cepillado dental en niños menores de tres años que en los niños mayores de esta edad; y en Turquía, Efe y colaboradores. ${ }^{7}$ encontraron que el nivel educativo de las madres tiene un efecto sobre el conocimiento de los niños en salud bucal. En Usaquén-Bogotá, Colombia, Martignon y colaboradores ${ }^{8}$ encontró que la mayoría de la población (80\%) sabe por qué se produce la caries y que se puede prevenir a través del cepillado dental. Además, $66 \%$ de la población inició la higiene bucal de los niños desde que aparecieron los dientes. Asimismo, en Medellín, Colombia, Franco y colaboradores ${ }^{9}$ obtuvo que $42 \%$ de las madres de estrato medio / alto y $50 \%$ del bajo afirmaran no haber recibido información sobre el cuidado bucal de sus hijos.

Este tipo de trabajos es pertinente debido a que permite el desarrollo de modelos innovadores de intervención preventiva en poblaciones vulnerables, teniendo en cuenta que los programas de salud bucal se han desa- rrollado con métodos y contenidos muy generales sin tener en cuenta la información recolectada previamente, por lo que se hace indispensable intentar en un futuro la construcción de nuevos juicios y actitudes saludables en las familias de los niños menores de cinco años y en sus cuidadores, para que contribuyan a disminuir las enfermedades bucales desde la infancia. ${ }^{10}$

En este sentido, el objetivo de este estudio fue describir los conocimientos, actitudes y prácticas frente a la problemática de salud bucal de los padres y cuidadores del Instituto Colombiano de Bienestar Familiar de la ciudad de Cartagena, Colombia.

\section{Material y métodos}

Diseño de estudio con enfoque mixto (cuantitativocualitativo), realizado en una población de 3450 padres y 23 cuidadores de niños de los hogares infantiles del Instituto Colombiano de Bienestar Familiar de Cartagena, Colombia (ICBF), durante el año 2010. Para el enfoque cuantitativo se utilizó una encuesta estructurada y para el enfoque cualitativo el método fueron los grupos focales.

El tamaño calculado de la muestra de padres fue de 333 , con un intervalo de confianza de $95 \%$, error del estudio de $5 \%$, poder de $80 \%$ y una frecuencia esperada del evento (buenos conocimientos) de $60.0 \%$. Además, la muestra de cuidadores fue de ocho, tomando un cuidador como representante de cada hogar seleccionado.

El muestreo de padres se realizó en forma probabilística por conglomerados. Inicialmente se identificaron 23 hogares infantiles en las tres localidades en que está distribuida la ciudad de Cartagena, según el actual plan de ordenamiento territorial (POT 2008). Fueron seleccionados aleatoriamente ocho hogares en la ciudad, teniendo en cuenta que no presentaran la modalidad de madres sustitutas y madres comunitarias (en esta modalidad se excluyeron 10 institutos, debido a que los niños permanecen todo el tiempo dentro de los hogares y no están en contacto con sus padres). A partir de cada conglomerado muestreado se realizó una selección aleatoria, permitiendo que el número de hogares representara a las tres localidades de la ciudad de Cartagena (Industrial de la Bahía 29.0\%, histórica $47.0 \%$ y de la Virgen 24.0\%). Posteriormente fueron muestreados los padres a través del método probabilístico simple por reemplazo, proporcional al tamaño de cada hogar. Los cuidadores fueron seleccionados a partir de un muestreo no probabilístico en forma intencional, incluyendo al cuidador responsable de los niños el día de la visita en los ocho hogares participantes.

Previo a la selección, se tuvo en cuenta el cumplimiento de los siguientes criterios: padres con hijos 
entre 1 y 5 años y cuidadores que estuvieran vinculados al ICBF de la ciudad de Cartagena, que aceptaran participar y gestionaron su consentimiento por escrito. Además, se excluyeron dos padres con dificultades motrices, diez padres que delegaban la responsabilidad del niño durante el día en otras personas y tres padres que se negaron a participar en el estudio.

El protocolo de la investigación fue sometido a la evaluación del Comité de Ética de la Universidad de Cartagena y la participación de los padres y cuidadores estuvo regulada por las normas éticas estipuladas en la declaración de Helsinki, modificación de Edimburgo 2000 y las normas científicas, técnicas y administrativas para la investigación en seres humanos, resolución 8430 de 1993 ministerio de salud, república de Colombia. Asimismo, los investigadores asumieron el compromiso de analizar y socializar los datos en forma global, sin violar la intimidad de los participantes.

\section{Instrumentos}

Se aplicaron dos tipos de instrumentos: encuestas estructuradas tipo cuestionario dirigidas a los padres de los niños que asisten a los hogares seleccionados y entrevistas individuales semiestructuradas y de grupos focales dirigidas a cuidadores. Los dos formatos medían los siguientes tópicos y categorías de análisis: visitas al odontólogo, higiene bucal, cepillado de dientes, uso de crema dental y alimentación (en este último aspecto se preguntó sobre el consumo de alimentos y bebidas endulzadas y uso de biberón por las noches). Al mismo tiempo se midieron variables sociodemográficas como la edad y el sexo de los participantes, el estrato socioeconómico (el cual fue valorado en tres categorías: bajo, 1 y 2; medio, 3 y 4; alto, 5 y 6, según el nivel de estratificación social que adquiere una persona a través de la ubicación de su vivienda, los servicios públicos y la actividad laboral [Planeación Nacional, República de Colombia, 2008]), la localidad en que se encuentra ubicado el hogar según las diferentes zonas de la ciudad de Cartagena (POT Cartagena de Indias 2008), el nivel educativo del padre entrevistado (medido a través del último grado de escolaridad obtenido) y régimen de salud al que pertenece el núcleo familiar (obtenido a partir de la afiliación al actual Sistema Nacional de Seguridad Social en Salud, Colombia, Ley 100 de 1993). El formato de encuesta fue diseñado por el grupo de la Unidad de Investigación en Caries, Universidad El Bosque UNICA, y validado en un estudio realizado por Martignon y Bautista, ${ }^{11}$ donde se obtuvo un alfa de Cronbach para conocimientos (0.82), actitudes $(0.85)$ y prácticas (0.62). El instrumento contiene 25 preguntas de conocimientos a partir de la siguiente clasificación: escaso: 0-33; aceptable: 34-66 y bueno: 67-100; 14 preguntas de actitudes con 26 ítems, escala de medición tipo Likert de 5 puntos, con rango de puntaje/sujeto de 26-130 puntos, con clasificación de actitud: desfavorable, 26-60; aceptable, 61-95, y favorable, 96-130; 28 preguntas de prácticas con 30 ítems.

De igual forma se diseñó una guía de entrevista individual semiestructurada dirigida a los cuidadores con 18 preguntas, tendientes a obtener una información dialógica a través de la interacción entre los cuidadores y los investigadores. Además, para complementar esta información se diseñó una guía temática de grupos focales con ocho preguntas, lo cual permitió complementar la información obtenida previamente a través de las entrevistas individuales, en unidades de análisis mucho más específicas dentro de la categorización inicial.

Antes de iniciar la recolección de los datos, los instrumentos (entrevistas y encuestas) fueron evaluados por dos jueces expertos para obtener validez de apariencia, luego se aplicaron diez encuestas en una muestra piloto en individuos con características similares a la población objeto, con el fin de evaluar las diferentes puntuaciones obtenidas con respecto a la teoría. Los resultados permitieron estandarizar a los encuestadores en el manejo del cuestionario y calibrar el instrumento. De igual forma, las entrevistas individuales y la guía temática para los grupos focales se aplicaron previamente en dos cuidadores con el fin de explorar la información conceptual concebida a priori por los investigadores, afinar la guía de entrevista, analizar el grado de profundidad de la información obtenida y corroborar el grado de comprensión de cada una de las preguntas en los potenciales participantes. En el proceso de recolección de información cualitativa siempre se utilizaron tres fases o momentos (exploración, focalización y profundización).

\section{Análisis estadístico}

La información del enfoque cuantitativo fue organizada y depurada a través de una base de datos en Excel 2007, luego fue analizada e interpretada en el programa estadístico STATA X. Los datos se analizaron a través de distribuciones de frecuencia y proporciones, calculados según el diseño muestral, asumiendo intervalos de confianza de $95 \%$. Para establecer relaciones entre conocimientos, actitudes y prácticas con las variables sociodemográficas se utilizó la prueba $\chi^{2}$ asumiendo un límite de 0.05 para la significancia. Además se realizó análisis multivariable por regresión logística nominal para variables confusoras (edad, sexo y estrato socioeconómico), siendo construida la variable dependiente a partir de dos indicadores; bueno contra escaso/acep- 
table para conocimientos y favorable contra aceptable/ desfavorable para actitudes. En el modelo se incluyeron aquellas variables que presentaron significancia igual o menor a 0.05 . La información del enfoque cualitativo se interpretó a través de una codificación abierta, realizándose una lectura detallada de los datos obtenidos con el fin de agrupar detalles y reformular preguntas, posteriormente se realizó una codificación selectiva redefiniéndose las categorías de análisis y confrontándose con la teoría (origen social de las enfermedades). Todo esto fue desarrollado a través del programa Atlas-Ti, a partir de discusiones de las diferentes categorías y de comentarios triangulados entre los investigadores y entre las unidades de análisis, ajustando con cada información la guía de entrevista mediante un modelo de análisis flexible típico de este enfoque, buscando patrones, discrepancias o similitudes entre las respuestas obtenidas, hasta lograr la saturación de la información.

\section{Resultados}

Los 333 padres participantes en el presente estudio tuvieron un promedio de edad de 35 años ( $\mathrm{DE}=3.8$ ). En cuanto a los niños, el promedio de edad fue de 2.9 años (DE=1.0), siendo los niños de 3 años los de mayor frecuencia con el $37.0 \%$. En cuanto al sexo, los masculinos fueron $51.0 \%$ y los femeninos $49.0 \%$. Con respecto al estrato socioeconómico, el más frecuente fue el uno (nivel bajo) con $45.0 \%$ y la localidad que más padres aportó fue la histórica con $47.0 \%$. Con respecto a los ocho hogares seleccionados, el que más unidades de estudio aportó fue el hogar "Las gaviotas" con 21.3\%. Con relación al régimen de salud al que se encontraban afiliados en núcleo familiar, el contributivo fue el de mayor frecuencia, con $51.0 \%$. Por último, el nivel de escolaridad más frecuente de los padres fue la secundaria completa, con $55.5 \%$. Con respecto a los cuidadores participantes en el estudio, tenían un promedio de edad de 42 años (DE=2.3).

\section{Análisis cuantitativo}

De acuerdo a los conocimientos observados en los participantes del presente estudio, la mayor frecuencia fue para el nivel bueno con 58.9\% (IC 95\%; 53.5-64.1). Con respecto a las actitudes, la mayor frecuencia fue para los valores favorables con 74.5\% (IC 95\%; 69.7-79.7).

En cuanto a las prácticas, el motivo de consulta al odontólogo de menor frecuencia fue por dolor o una urgencia dental para los padres (19.6\%; IC14.2-25.0) y los niños (6.6\%; IC3.3-10.0). En este aspecto se encontró una alta frecuencia de padres (51.3\%; IC44.4-58.1) y niños (68.8\%; IC61.5-74.0) que estuvieron totalmente en desacuerdo en visitar al odontólogo solo por esta causa y resaltaron otras más recomendables como control y prevención (46.2\%; IC39.4-53.0) (cuadro I). $\mathrm{Al}$ indagar por la edad de inicio de la higiene bucal en los niños, 33.3\% (IC24.7-37.3) de los padres manifestaron que la iniciaron en sus hijos desde el nacimiento, por otra parte, 68.4\% (IC62.1-74.6) considera que ellos son los responsables de su higiene bucal. En cuanto al interrogante de si los niños se van a dormir con los dientes cepillados, 50.6\% (IC43.8-57.4) de los padres informaron que éstos siempre realizan dicha actividad y $69.6 \%$ (IC63.4-75.8) afirman que son ellos quienes colocan la crema dental sobre el cepillo. Por otro lado, al preguntar si la cantidad de crema dental apropiada para el cepillado del niño es menos de la mitad del largo del cepillo dental, la mayor parte de los encuestados estuvo totalmente en desacuerdo (66.4\%) (cuadro I).

En el análisis bivariado se observaron relaciones con significancia estadística entre los niveles de conocimientos y el estrato socioeconómico $(p=0.05)$; los padres que pertenecen al nivel medio/ alto (tres, cuatro $\mathrm{y}$ cinco) fueron los que obtuvieron los mejores valores $48 / 71(67.6 \%)$, con respecto a los del nivel bajo (uno y dos) 148/262 (56.5\%). En cuanto a los hogares, el mayor porcentaje de padres con buenos conocimientos se presentó en el hogar "Las gaviotas" con 91.6\%, en comparación con "El pescador" (38.5\%) y "Santa Rita" $(40.5 \%)(p=0.000)$. De acuerdo con la localidad, en la Industrial de la Bahía se observaron buenos conocimientos en $80.4 \%$, con respecto a la histórica y La Virgen $(p=0.000)$. Además fue significativa la relación con el régimen de salud al que estaban vinculados los padres, con el contributivo el de mayores valores (66.5\%), en comparación con el subsidiado (50.1\%); $p=0.02)$. Para las demás relaciones no hubo significancia estadística (cuadro II).

En cuanto a la relación entre actitudes y variables sociodemográficas, se encontró significancia estadística con el sexo ( $p=0.04)$; los individuos de sexo masculino tuvieron mejores actitudes favorables $(79.3 \%)$. Además hubo significancia con los hogares evaluados; en "Las gaviotas" se encontró la mayor frecuencia de actitudes favorables (87.3\%), en comparación con "El pescador" $(57.7 \%)$ y "Los ciruelos" (63.3\%) ( $p=0.02)$. Con respecto al régimen de seguridad social y el nivel de escolaridad, se observaron mejores actitudes en el subsidiado $(79.6 \%$; $p=0.03)$ y en el nivel de escolaridad medio / alto (75.6\%; $p=0.03$ ) (cuadro III).

En lo referente a la relación entre prácticas y estrato socioeconómico, se obtuvo una mayor frecuencia de padres de estrato bajo (uno y dos), que siempre realizan la higiene bucal de sus hijos (79.5\%), en comparación con los 


\section{Cuadro I}

Frecuencia de prácticas de los padres para la salud oral en los niños ICBF, Cartagena, Colombia 2010

\begin{tabular}{|c|c|c|c|c|c|c|c|}
\hline & $\begin{array}{c}\text { Número } \\
n=333\end{array}$ & $\begin{array}{c}\text { Frecuencia } \\
(\%)^{*}\end{array}$ & IC $95 \%$ & & $\begin{array}{l}\text { Número } \\
n=333\end{array}$ & $\begin{array}{c}\text { Frecuencia } \\
(\%)^{*}\end{array}$ & IC $95 \%$ \\
\hline I.Visita al odontólogo/padres & & & & 4.Acerca de la crema dental & & & \\
\hline Consulta por dolor o urgencia & & & & El padre la coloca en el cepillo _ & & & \\
\hline Definitivamente sí & 64 & 19.6 & $14.2-25.0$ & Siempre & 235 & 69.6 & $63.4-75.8$ \\
\hline Probablemente sí & 22 & 6.6 & $3.2-10.1$ & Casi siempre & 50 & 14.9 & $10.1-19.7$ \\
\hline No recuerda & 37 & 11.7 & $7.3-16.1$ & No sabe & 7 & 2.5 & $0.2-4.8$ \\
\hline Probablemente no & 29 & 10.8 & $6.4-15.1$ & Casi nunca & 12 & 3.5 & $1.2-5.7$ \\
\hline Definitivamente no & 181 & 51.3 & 44.4-58.I & Nunca & 29 & 9.5 & $5.4-13.6$ \\
\hline 2.Visita al odontólogo/niños & & & & Utiliza menos de la mitad del cepillo & & & \\
\hline Consulta por dolor/urgencia & & & & Totalmente de acuerdo & 113 & 39.9 & $33.2-46.6$ \\
\hline Definitivamente sí & 22 & 6.6 & $3.3-10.0$ & De acuerdo & 109 & 30.7 & $24.4-37.0$ \\
\hline Probablemente sí & 18 & 6.6 & $3.2-10.1$ & Neutral & 89 & 22.1 & 16.6-27.7 \\
\hline No recuerda & 29 & 10.1 & $6.1-14.2$ & En desacuerdo & 12 & 4.1 & $1.6-6.6$ \\
\hline Probablemente no & 37 & 8.9 & $5.3-12.4$ & Totalmente en desacuerdo & 10 & 3.2 & $\mid .2-5.1$ \\
\hline Definitivamente no & 227 & 68.8 & $61.5-74.0$ & 5. Higiene bucal de niños & & & \\
\hline Consulta por control/prevención & & & & Inicio de la higiene oral & & & \\
\hline Definitivamente sí & 158 & 46.2 & $39.4-53.0$ & Desde que nació & 111 & 31.0 & $24.7-37.3$ \\
\hline Probablemente sí & 33 & 9.5 & $5.5-13.4$ & $\begin{array}{l}\text { Desde que le erupcionaron los } \\
\text { dientes }\end{array}$ & 87 & 25.6 & $19.7-3 \mid .6$ \\
\hline No recuerda & 24 & 7.9 & $4.2-11.7$ & De I a 2 años & 84 & 247 & $188-306$ \\
\hline Probablemente no & 17 & 4.4 & I.7-7.2 & & & $2 \ldots$ & 10.050 \\
\hline Definitivamente sí & 101 & 3.2 & $2.6-3.8$ & De 2 a 3 anos & 34 & 12.7 & $8.1-1 / .3$ \\
\hline 3.Alimentación & & & & Mayor de 3 años & 17 & 6.1 & $2.9-9.2$ \\
\hline Consume alimentos endulzados & & & & Los padres realizan la higiene & & & \\
\hline Siempre & 145 & 47.2 & $40.3-54.0$ & Siempre & 224 & 68.4 & $62.1-74.6$ \\
\hline Casi siempre & 119 & 35.1 & $28.6-41.6$ & Casi siempre & 71 & 21.5 & $16.0-27.0$ \\
\hline No sabe & 13 & 3.8 & $0.9-6.6$ & No sabe & 4 & 1.3 & $0.2-2.8$ \\
\hline Casi nunca & 48 & II.4 & $7.3-15.5$ & Casi nunca & 22 & 5.1 & 2.5-7.7 \\
\hline Nunca & 8 & 2.5 & $0.6-4.5$ & Nunca & 12 & 3.8 & $1.2-6.4$ \\
\hline Práctica de biberón en la noche & & & & Cepillado antes de dormir & & & \\
\hline Totalmente de acuerdo & 55 & 174 & $122-276$ & Siempre & 169 & 50.6 & $43.8-57.4$ \\
\hline & $\Delta !$ & 95 & 56,134 & Casi siempre & 101 & 29.7 & $23.5-35.9$ \\
\hline Neutral & 31 & 87 & 46118 & No sabe & 6 & 1.9 & $0.5-3.8$ \\
\hline En desacuerdo & 64 & 212 & $155-269$ & Casi nunca & 36 & 9.5 & $5.5-13.4$ \\
\hline Totalmente en desacuerdo & 42 & 437 & 369,504 & Nunca & 21 & 8.2 & $4.4-12.1$ \\
\hline Total & 333 & 100.0 & & Total & 333 & 10.0 & \\
\hline
\end{tabular}

* Estimadores ajustados por diseño muestral 
Cuadro II

RELACIÓN ENTRE NIVEL DE CONOCIMIENTOS DE LOS PADRES Y VARIABLES SOCIODEMOGRÀFICAS en Cartagena, Colombia 2010

\begin{tabular}{|c|c|c|c|c|c|c|}
\hline & Bueno $n=196$ & $(\%)^{*}$ & Aceptable/escaso $n=137$ & $(\%)^{\ddagger}$ & Total & Valor $p$ \\
\hline \multicolumn{7}{|l|}{ Edad } \\
\hline $1-2$ & 67 & 58.3 & 48 & 41.7 & 115 & \\
\hline $3-5$ & 129 & 59.2 & 89 & 40.1 & 218 & 0.87 \\
\hline \multicolumn{7}{|l|}{ Sexo } \\
\hline Masculino & 105 & 62.1 & 64 & 37.9 & 169 & \\
\hline Femenino & 91 & 55.5 & 73 & 44.5 & 164 & 0.21 \\
\hline \multicolumn{7}{|c|}{ Estrato socioeconómico } \\
\hline Bajo $(I, 2)$ & 148 & 56.5 & 114 & 43.5 & 262 & \\
\hline Medio/Alto $(3,4,5)$ & 48 & 67.6 & 23 & 32.4 & 71 & $0.05 \S$ \\
\hline \multicolumn{7}{|l|}{ Localidad } \\
\hline Industrial & 78 & 80.4 & 19 & 19.6 & 97 & \\
\hline Histórica & 80 & 51.3 & 76 & 48.7 & 156 & $0.000^{\S}$ \\
\hline De la Virgen & 38 & 47.5 & 42 & 52.5 & 80 & \\
\hline \multicolumn{7}{|l|}{ Hogar Infantil } \\
\hline Las Gaviotas & 65 & 91.6 & 6 & 8.5 & 71 & \\
\hline El Labrador & 22 & 52.4 & 20 & 47.6 & 42 & \\
\hline España & 20 & 42.6 & 27 & 57.6 & 47 & \\
\hline Santa Rita & 15 & 40.5 & 22 & 59.5 & 37 & $0.000^{\S}$ \\
\hline El Pescador & 10 & 38.5 & 16 & 61.5 & 26 & \\
\hline Don Blas & 28 & 51.9 & 26 & 48.1 & 54 & \\
\hline Los Ciruelos & 23 & 76.8 & 7 & 23.3 & 30 & \\
\hline San Lucas & 13 & 50 & 13 & 50 & 26 & \\
\hline \multicolumn{7}{|l|}{ Régimen de salud } \\
\hline R. Subsidiado & 84 & 50.1 & 80 & 49.1 & 163 & \\
\hline R. Contributivo & 112 & 66.5 & 57 & 33.5 & 170 & $0.02^{\S}$ \\
\hline \multicolumn{7}{|c|}{ N. Educativo de padres } \\
\hline Bajo & 9 & 52.9 & 8 & 47.1 & 17 & \\
\hline Medio/alto & 187 & 59.2 & 129 & 40.8 & 316 & 0.61 \\
\hline
\end{tabular}

* Estimadores sin ajustar para el evento de estudio favorable; ${ }^{\ddagger}$ estimadores sin ajustar para el evento de estudio desfavorable; ${ }^{\S}$ valores de probabilidad con significación estadística.

padres de estrato medio/ alto (tres, cuatro y cinco) (20.5\%; $p=0.04)$ ). Para las demás variables no se encontró significancia. También se analizó la distribución de las prácticas de higiene bucal en la población de estudio con respecto al régimen de afiliación al Sistema Nacional deSeguridad Social en Salud, y se observó una mayor frecuencia de padres que colocan la crema dental sobre el cepillo de sus hijos y pertenecen al régimen contributivo $(51.9 \%)$ con relación a los padres afiliados al régimen subsidiado (48.1\%), pero la diferencia no fue significativa.
En relación con la frecuencia del consumo dealimentos cariogénicos (dieta rica en azúcares y carbohidratos fermentables) y el nivel de escolaridad de los padres, se encontró mayor frecuencia de consumo en padres con escolaridad primaria y secundaria $(67.6 \%)$ respecto a los padres con escolaridad técnica y universitaria (32.4\%; $p=0.01)$.

Por último, en el análisis multivariable, el mejor modelo para niveles de conocimientos sobre higiene bucal se obtuvo con las variables sexo $(p=0.13)$, estrato socioeconómico 


\section{Cuadro III}

\section{ReLACIÓn ENTRE LAS ACTITUdeS EN LOS PADRES Y LAS VARIABLES SOCIODEMOGRÀFICAS, Cartagena, Colombia 2010}

\begin{tabular}{|c|c|c|c|c|c|c|}
\hline & Favorable $n=248$ & $(\%)^{*}$ & Acept/desfavorable $n=85$ & $(\%)^{\ddagger}$ & total & Valor $p$ \\
\hline \multicolumn{7}{|l|}{ Edad } \\
\hline I- 2 años & 81 & 70.4 & 34 & 29.6 & 115 & \\
\hline 3- 5 años & 167 & 70.6 & 51 & 23.4 & 218 & 0.21 \\
\hline \multicolumn{7}{|l|}{ Sexo } \\
\hline Masculino & 134 & 79.3 & 35 & 20.7 & 169 & \\
\hline Femenino & 114 & 69.5 & 50 & 30.5 & 164 & $0.04^{\S}$ \\
\hline \multicolumn{7}{|l|}{ Localidad } \\
\hline Industrial de la Bahía & 81 & 83.5 & 16 & 16.5 & 97 & \\
\hline Histórica & 110 & 70.5 & 46 & 29.5 & 156 & 0.05 \\
\hline De la Virgen & 57 & 71.3 & 23 & 28.8 & 80 & \\
\hline \multicolumn{7}{|l|}{ Hogar infantil } \\
\hline Las Gaviotas & 62 & 87.3 & 9 & 12.7 & 71 & \\
\hline El Labrador & 27 & 64.3 & 15 & 35.7 & 42 & \\
\hline España & 34 & 72.3 & 13 & 27.7 & 47 & \\
\hline Santa Rita & 30 & 81.1 & 7 & 18.9 & 37 & $0.02^{\S}$ \\
\hline El Pescador & 15 & 57.7 & 11 & 42.3 & 26 & \\
\hline Don Blas & 42 & 77.8 & 12 & 22.2 & 54 & \\
\hline Los Ciruelos & 19 & 63.3 & 11 & 36.7 & 30 & \\
\hline San Lucas & 19 & 73.1 & 7 & 26.9 & 26 & \\
\hline \multicolumn{7}{|l|}{ Régimen de salud } \\
\hline Subsidiado & 129 & 79.6 & 33 & 20.4 & 164 & \\
\hline Contributivo & 119 & 69.6 & 52 & 30.4 & 169 & $0.03^{\S}$ \\
\hline \multicolumn{7}{|c|}{ Nivel de escolaridad de padres } \\
\hline Bajo & 9 & 52.9 & 8 & 47.1 & 17 & \\
\hline Medio/Alto & 239 & 75.6 & 77 & 24.4 & 316 & $0.03^{\S}$ \\
\hline
\end{tabular}

$(p=0.000)$, localidad $(p=0.03)$ y hogar infantil $(p=0.18)$; $\left(\chi^{2}=41.7 ; p=0,000\right)$, mientras que para las actitudes hacia a la higiene oral, el mejor modelo se observó con el sexo $(p=0.07)$, hogar infantil $(p=0.03)$, seguridad social $(p=0.03)$ y nivel de escolaridad $(p=0.02) ;\left(\chi^{2}=15.4\right.$; $p=0,003$ ) (cuadro IV).

\section{Análisis cualitativo}

En lo que tiene que ver con las percepciones de los cuidadores, se obtuvo información a partir de las siguientes unidades de análisis. En lo que se refiere a la etiología de la enfermedad bucal, a la pregunta ¿A qué le atribuye que la población infantil en edades menores de cinco años se vean afectados por enfermedades bucales como la caries dental?, los cuidadores indicaron a los padres como los principales responsables de que en los menores se presenten patologías bucales, debido a que son ellos quienes deben estar atentos de la higiene bucal de los niños, ya que en esta etapa no son autónomos y no tienen la destreza para realizar estos procedimientos. Además, se considera que éstos deben ser responsables de la asistencia odontológica frecuente del menor, a fin de atender tempranamente cualquier indicio de enfermedad, o de 


\section{Cuadro IV}

Modelo multivariable para conocimientos y ACtitudes de los PADRes hacia la higiene ORAL DE ACUERDo CON variables sociodemogràficas, Cartagena, Colombia 2010

\begin{tabular}{llccc} 
Conocimiento de padres* & Coef & Error & Valor $p$ & IC $95 \%$ \\
Sexo masculino & 0.35 & 0.23 & 0.13 & $-0.11-0.82$ \\
\hline Estrato socioeconómico bajo & -0.66 & 0.16 & 0.000 & $-0.99--0.33$ \\
\hline Localidad de la Virgen & 0.39 & 0.18 & 0.03 & $0.02-0.75$ \\
\hline Hogar infantil El Pescador & 0.07 & 0.05 & 0.18 & $-0.03-0.18$ \\
Actitudes de padres & & & 0.07 & $-0.04-0.97$ \\
Sexo masculino & 0.47 & 0.26 & $-0.02-0.20$ \\
\hline Hogar infantil El Pescador & 0.09 & 0.05 & 0.10 & $0.04-0.99$ \\
\hline Seguridad social contributivo & 0.57 & 0.27 & 0.03 & $-2.2--0.14$
\end{tabular}

Significancia estadística del modelo a partir de variables con valores $p<=0.05$ $*(\chi 2=41.7 ; p=0.000) ;{ }^{\ddagger}(\chi 2=15.4 ; p=0.003)$.

buscar orientación en dicho evento si se desconoce. Se encontraron expresiones como "una de las razones que yo intuyo para este problema es que los padres a esta corta edad no le prestan atención a la importancia de la salud bucal en sus hijos, no les hacen los controles respectivos, siendo estas las principales causas para la aparición de la caries dental, aquí nosotros ahora para la matricula les exigimos a los padres un certificado de salud bucal, para que ellos se vean en la obligación de llevar a los niños y así mirar la parte física y la parte de la salud bucal".

Con respecto a la segunda categoría -medidas adecuadas para prevenir o disminuir las enfermedades bucales-, los cuidadores consideran que la educación al padre de familia es la medida más importante que se debe instaurar, debido a que ellos son los encargados de educarlos y a través de esto se pueden ir creando hábitos de higiene bucal desde muy temprana edad. Se parte del concepto "la educación empieza en el hogar", a través de programas que capaciten al padre de familia en hábitos de higiene bucal saludables para que los ayuden a llevar un control adecuado de la limpieza después de alimentarlos y prepararlos para que sean conscientes de la asistencia odontológica. En este sentido se encontraron descripciones como "Se debe concientizar a los padres de familia y a los niños de lo importante que es mantener una higiene bucal adecuada, que al igual que asearse el cuerpo es importante asearse los dientes. También que así como se asiste al médico hay que asistir al odontólogo, educando a los padres con charlas y haciendo énfasis de que lleven a los niños a controles".

Por otro lado, se percibe a partir de muchas opiniones de los cuidadores que el estrato socioeconómico no es un factor influyente en la salud bucal de los niños, pues según los participantes, "La enfermedad oral se trata de hábitos que se deben aprender en casa y esto no tiene ningún costo, además, actualmente con tantos programas de salud que tiene el gobierno, es triste que se pueda presentar este problema, pues creo que hay muchas oportunidades para las personas de bajos recursos a que acudan a centros de salud y mantengan un buen control odontológico". Se considera además que la información que se tenga puede influir en cierto grado, pues es probable que aquellos padres que han recibido capacitación sobre el cuidado de las estructuras bucales tengan mejores conocimientos, actitudes y prácticas que puedan transmitir a sus niños.

Con respecto a la tercera categoría -actividades de higiene bucal que se realizan dentro de los hogares infantiles-, según las opiniones de los cuidadores se consideran efectivas, debido a que éstas contribuyen a la promoción de la salud y prevención de las enfermedades y mantienen una actitud favorable hacia la higiene. Una de las acciones más importantes evidenciadas dentro de cada hogar infantil es la exigencia de un examen odontológico para el ingreso del niño y un control cada año, así como también la implementación de un programa llamado "Escuela para padres" lo cual fomenta la educación y la creación de hábitos de higiene bucal en sus hijos con la realización del cepillado cada vez que ingieren algún tipo de alimento dentro del hogar.

En general los cuidadores manifiestan que los niños muestran actitudes positivas en el momento en que realizan las prácticas de higiene bucal como el cepillado dental, les gusta y lo disfrutan, pero cuando se 
trata de visitar al odontólogo la mayoría siente temor porque lo asocian con dolor o molestia. Se considera que los especialistas en salud bucal son las principales fuentes de conocimientos en cuanto al cuidado de las estructuras bucales, por lo tanto son ellos los encargados de propender por la preservación de la boca y realizar la promoción y prevención necesarias. Por otra parte también es importante la labor que realizan los cuidadores, pues los niños pasan gran parte de su tiempo en el hogar infantil y mientras ellos estén bajo su cuidado son los responsables de su educación.

\section{Discusión}

Si bien los hallazgos del presente estudio provienen de una información transversal obtenida a partir de una encuesta y dos formatos de entrevistas, en el diseño se lograron incluir los elementos mínimos requeridos para la obtención de validez: utilización de instrumentos validados en la literatura y confirmados en prueba piloto, obtención de una muestra probabilística proporcional al tamaño de cada localidad de la ciudad de Cartagena, restricción de sujetos a partir de criterios de exclusión y análisis estratificado.

Los resultados obtenidos se consideran importantes, ya que para que una intervención tenga éxito es necesario conocer previamente las necesidades reales del grupo poblacional al cual va dirigida, tratando de enfocarse en los aspectos que influyen en la naturaleza misma de la enfermedad, los cuales pueden ser analizados desde la perspectiva de los padres y cuidadores para servir de guía a los profesionales y tenerlos en cuenta para preservar la salud bucal en estos individuos. ${ }^{12}$

Con respecto a los conocimientos de los padres, se encontró que más de la mitad presentaban buenos niveles, lo que se demostró en preguntas tales como ¿Por qué se produce la caries dental?, ¿Cuál es la mejor manera de evitarla? y ¿Cuál es el momento adecuado para el uso de la crema dental? Estos hallazgos son más sobresalientes que los reportados en el ámbito internacional. En Malasia, Mani ${ }^{13}$ encontró que sólo 32\% de los padres eran conscientes de que la caries dental puede afectar en la dentición temporal y $38 \%$ conoce la edad correcta de inicio del uso de dentífricos. En estudios realizados en Colombia, Jiménez y colaboradores ${ }^{14}$ en ciudades como Bogotá, Cartagena, Manizales y Medellín, encontraron que $98.0 \%$ de la población encuestada consideró importante cuidar los dientes primarios.

En cuanto a las actitudes, en el presente estudio también se observaron resultados favorables en un alto porcentaje, similares a los reportados por Vanagas ${ }^{15}$ en Lituania, donde la mayor parte de los padres mostraron tendencias positivas con respecto a la dieta y la higiene bucal de sus hijos ( $p=0.001$ y $p=<0.001$, respectivamente).

Dentro de las prácticas, un alto porcentaje de los encuestados estuvo totalmente en desacuerdo con que la cantidad apropiada de crema dental para cepillar al niño fuese menor de la mitad del largo de las cerdas del cepillo. Estos hallazgos son similares a lo encontrado por González y colaboradores ${ }^{16}$ en niños de la ciudad de Cartagena, Colombia, que observaron que $44 \%$ de padres manifestaron utilizar como parámetro apropiado entre la mitad del cepillo y el cepillo completo. Asimismo, Jassem, ${ }^{17}$ en Kuwait, encontró que 55\% de la población evaluada utiliza como cantidad de dentífrico más de la mitad del cepillo. De esta forma se reconoce que este tipo de conductas resulta un factor predisponente para la aparición de fluorosis dental, pues es en este intervalo de edades (2-5 años) donde la dentición permanente se encuentra en un período crítico de calcificación ${ }^{18}$ y en la que los niños ingieren mayor cantidad de crema de la que expectoran. ${ }^{19}$

Otro aspecto importante en relación con esta problemática es la supervisión del cepillado del niño, que es una conducta de riesgo para aquellos que realizan esta actividad sin el monitoreo de los padres. El presente estudio mostró resultados positivos, debido a que la mayoría de los padres siempre realizan la higiene bucal de sus hijos, siendo más frecuente esta conducta en los que pertenecen a estratos bajos. Además, la mayoría de los padres son quienes colocan la crema dental sobre el cepillo del niño. En contraste Martignon y colaboradores $^{8}$ en Usaquén-Bogotá, Colombia, informa de una mayor frecuencia del monitoreo del cepillado dental en los padres de estrato medio (tres). Franco y colaboradores ${ }^{9}$ en Medellín, Colombia, encontraron que tan solo un pequeño porcentaje de niños dispensaban por sí mismos el dentífrico sobre el cepillo.

Por otra parte, en este estudio un poco menos de la mitad de los padres estuvieron totalmente en desacuerdo con la práctica del biberón por las noches para la alimentación de sus hijos, sobre todo cuando se trata de un contenido diferente al agua o el uso de un chupón endulzado, lo cual es comparable con lo expuesto por Schroth $^{20}$ en Manitoba, Canadá, quien informó que los niños cuyos cuidadores estaban de acuerdo con la alimentación con biberón después del primer año tuvieron índices de higiene bucal más deficientes que aquellos que consideraban lo contrario $(4.8 \pm 5.2$ frente a $3.7 \pm$ $4.8, p=0.0015)$.

En lo concerniente a las visitas al odontólogo por parte de padres y niños, el motivo de consulta de menor frecuencia en el presente estudio fue por urgencias en casos de dolor dental, siendo otras causas las de mayor prioridad, como en el caso de control y prevención. Estos 
resultados son similares a lo reportado por Saldarriaga y colaboradores ${ }^{21}$ en Medellín, Colombia en 2003, donde estos últimos motivos de consulta se observaron en el $48.6 \%$ de los padres, pero diferente a lo encontrado por Farsi y colaboradores ${ }^{22}$ quien observó entre todo el grupo evaluado que el dolor es la razón principal para visitar al odontólogo.

Al indagar las percepciones de los cuidadores sobre la importancia de la salud bucal en los niños, en el presente estudio se encontró una actitud positiva hacia el desarrollo de programas de promoción y prevención en el hogar infantil, algo que es significativo a la hora de la implementación de los mismos. Sin embargo, éstos coincidieron en afirmar que son los padres los principales responsables de guiar a los niños para la realización de una buena higiene bucal, a pesar de la ayuda que se les pueda suministrar. Escobar y colaboradores ${ }^{23}$ en Medellín, Colombia, encontraron que las madres se manifiestan dispuestas a dar a sus hijos una buena salud bucal, la cual consideran que es su responsabilidad, pero reclaman un mayor apoyo del personal de salud. Estos hallazgos permitieron evidenciar las dificultades que los cuidadores han encontrado para lograr una colaboración eficiente en las acciones educativas que se utilizan a diario dentro del hogar infantil.

En este mismo sentido, la percepción de los cuidadores en torno a las ventajas que ofrecen estas interacciones entre los diferentes actores del problema, pueden explicar la desmitificación que estos le dieron a la relación estrato socioeconómico e higiene bucal, lo que les permitió tener una explicación interesante, la cual es coherente con la cultura de los individuos y el empoderamiento que tiene un colectivo para transformar las oportunidades de acceso a los servicios de salud producto de las desigualdades sociales. ${ }^{24}$ Estos hallazgos son contrarios a los provenientes de la información de padres, los cuales demuestran a través de dos modelos de regresión, que las variables de mayor peso para explicar la presencia de buenos niveles de conocimientos sobre higiene bucal fueron el estrato socioeconómico y la localidad donde se encontró ubicado el hogar infantil y para actitudes favorables fueron el régimen de seguridad social y el nivel educativo de los padres. Estos aspectos han sido evaluados en numerosos estudios poblacionales ${ }^{25-27}$ en donde se ratifica que las condiciones de mayor vulnerabilidad social en las poblaciones, como el escaso acceso al trabajo, salud, vivienda y educación -los cuales se consideran factores influyentes para la obtención de hábitos higiénicos saludables-, se traducen en el deterioro de la salud bucal de los individuos. Desde esta perspectiva, se evidencian dos posiciones diametralmente opuestas provenientes de los dos principales actores de esta problemática, lo que podría ser visto por el grupo investigador como una oportunidad para seguir impulsando encuentros entre las partes y poder involucrar estas opiniones en un proceso de interacción tripartita (padres, cuidadores y niños), en quienes se reflejarían estas conclusiones para lograr el mantenimiento de la salud bucal desde tempranas edades.

Los resultados del presente estudio serían aplicables a la luz del diseño de intervenciones acordes con las necesidades de los individuos, lo que puede garantizar un impacto positivo sobre su salud oral, a partir de la consolidación de los conocimientos, promoción de actitudes favorables y prácticas orales adecuadas. Siendo la salud bucal un aspecto importante en el cuidado integral de la población infantil, los padres de familia se consideran la fuente primaria de la educación temprana en los menores, así como los maestros y toda persona encargada de su cuidado. Actualmente el número de padres que trabajan fuera del hogar va en incremento, por lo que los niños pasan una cantidad considerable de tiempo en centros educativos, de manera que su personal se involucra en la higiene general y el cuidado de la salud oral, de modo que todas las intervenciones de promoción deben ir dirigidas a ambos grupos.

La información obtenida en el presente estudio se complementa con el análisis de salud bucal realizado en esta misma población a inicios del periodo de evaluación, donde se identificaron las principales necesidades en salud bucal y se encontró una alta prevalencia de caries dental, a pesar de los buenos resultados observados en los conocimientos, actitudes y prácticas. Esto permite que se reflexione en torno a que no es suficiente el uso de un solo enfoque diagnóstico para tomar decisiones preventivas o terapéuticas dentro de un grupo de individuos.

A partir de los resultados obtenidos se concluye que los niveles satisfactorios de conocimientos, actitudes favorables y prácticas adecuadas observadas en el presente estudio y las interesantes percepciones de los participantes encaminadas a lograr una interacción entre los responsables del cuidado de los niños para afianzar el uso de herramientas de higiene bucal, puede ser visto como una oportunidad para el abordaje de poblaciones similares en regiones con características sociodemográficas y culturales típicas del caribe colombiano, a través de programas promocionales integrales que ayuden a mejorar la calidad de vida de los individuos desde tempranas edades, teniendo en cuenta su entorno familiar y social.

\section{Agradecimientos}

A la Universidad de Cartagena por todo el apoyo académico y administrativo durante la realización de este 
proyecto. Al ICBF seccional Cartagena por permitirnos el ingreso a cada uno de los hogares infantiles, así como a las coordinadoras de cada uno de ellos y sus docentes, quienes facilitaron el desarrollo de la recolección de los datos. A todos los padres encuestados, cuya información fue vital para la obtención de estos resultados.

Declaración de conflicto de intereses: Los autores declararon no tener conflicto de intereses.

\section{Referencias}

I. Ministerio de Salud -Colombia. Resolución número 00412 del 25 de febrero 2000. Normas Técnicas y Guías de Atención. Diario Oficial; 2000, Colombia.

2. González F, Sánchez R, Carmona L. Indicadores de riesgo para la caries dental en niños preescolares de la Boquilla, Cartagena. Rev salud pública 2009; II (4): 620-630.

3. Díaz S, González F. Prevalencia de caries dental y factores familiares en niños escolares de Cartagena de Indias, Colombia. Rev Salud Pública 2010; 12(5): $721-728$.

4. Ismail Al, Sohn W, Tellez M, Amaya A, Sen A, Hasson H, Pitts NB. The International Caries Detection and Assessment System (ICDAS): and integrated system for measuring dental Caries. Community Dent Oral Epidemiol 2006; 34: I-9.

5.Watson M, Horowitz A, Garcia I. Caries conditions among 2-5 years old inmigrant latino children related to parents oral health knowledge opinions and practices. Community dentistry and oral epidemiology 1999; 27(1):8-15

6. Finlayson TL, Siefert K, Ismail AI, Sohn W.Maternal self-efficacy and I-5year-old children's brushing habits. Community Dent Oral Epidemiology 2007; 272-81.

7. Efe E, Sarvan S, Kukulu K, Self-reported knowledge and behaviors related to oral and dental health in turkish children. Pediatric nursing 2007; 30:133-146.

8. Martignon S, González M, Jácome S, Velosa J, Santamaría R. Conocimientos, actitudes y prácticas en salud oral de padres y jardineras de niños hogares infantiles: ICBF - Usaquén, Bogotá. Revista científica Universidad El Bosque 2003; 9 (2):2003.

9. Franco A. Santamaría A. Kurzer E. Castro L. Giraldo M. el menor de seis años: situación de caries y conocimientos y prácticas de cuidado bucal de sus madres. Revista CES Odontología 2004; 17 (I):19-29.

10. Morrison J], Macpherson LMD, Binnie VI.A Qualitative Investigation of the Perceived Barriers to and Inducements for the Early Registration of Infants with General Dental Practitioners. Int J Health Prom \& Educ 2000; 38(I): 4-9.

I I. Martignon S, Bautista G, González M, Lafaurie G, Morales V, Santamaría R. Instruments for evaluating oral health knowledge, attitudes and practice for parents /caregivers of small children. Rev Salud pública 2008; 10(2): 308-314.
12. Southward LH, Robertson A, Wells-Parker E, Eklund NP, Silberman SL, Crall JJ, et al. Oral health status of mississippi delta 3- to 5-year-olds in child care: an exploratory study of dental health status and risk factors for dental disease and treatment needs.J Public Health Dent 2006; 66:131-7. 13. SA Mani,AA Aziz, J John, NM Ismail. Knowledge, attitude and practice of oral health promoting factors among caretakers of children attending day-care centers in Kubang Kerian, Malaysia:A preliminary study. Journal of Indian Society of Periodontics and Preventive Dentistry 2010;28(2):78-83. 14. Franco A, Jiménez J, Saldarriaga C, Zapata I, Saldarriaga A.

Conocimientos y prácticas de cuidado bucal de las madres de niños menores de seis años. Revista CES odontología 2004; 16 (3):23-34. I5.Vanagas G, Milasauskiene Z, Grabauskas V, Mickeviciene A.Associations between parental skills and their attitudes toward importance to develop good oral hygiene skills in their children. Medicina (Kaunas) 2009; 45(9):718-23.

16. González F, Carmona L, Díaz A. Percepción de ingesta de flúor a través del cepillado dental en niños colombianos. Revista Cubana de Estomatología 2010; 47(3):266-27.

17. Jassem A, Eino H, Sisko H. Oral health knowledge and behavior among male health sciences college students in Kuwait. BMC Oral Health 2003; 3: [Fecha de acceso 2008/I0/0I]. URL disponible en: http://www. biomedcentral.com/1472-683I/3/2.

18. Den Besten PK. Biological mechanisms of dental fluorosis relevant to the use of fluoride supplements. Community Dent Oral Epidemiol 1999; 27:41-7.

19. Rojas F, Kelly S, Drake K, Eckert G, Stookey G, Dunipace A. Fluoride intake from foods, beverages and dentifrice by young children in communities with optimally fluoridated water. Community Dent Oral Epidemiol 1999; 27:288-97.

20. Schroth RJ, Brothwell DJ, Moffatt ME.Canadian Child Health Clinican Scientist Program, Faculty of Dentistry, University of Manitoba, Caregiver knowledge and attitudes of preschool oral health and early childhood caries. ECC 2007 Apr; 66(2): 153-67.

21. Saldarriaga O, Sánchez M,Avendaño L. Conocimientos y prácticas en salud bucal de las gestantes vinculadas al programa de control prenatal. Medellín 2003. Revista CES Odontología 2004; 17(2): 34-44.

22. Farsi JM, Farghaly M, Farsi N. Oral health knowledge, attitude and behavior among Saudi school students in Jeddah city. J Dent 2004; 32(I): 47-53.

23. Escobar G, Sosa C, Burgos L. Representaciones sociales del proceso salud-enfermedad bucal en madres gestantes de una población urbana. Medellín, Colombia. Salud pública de México 2010; 52(I):46-5I.

24. Hilton IV, Stephen S, Barker JC, Weintraub JA. Cultural factors and children's oral health care: a qualitative study of carers of young children. Community Dent Oral Epidemiol 2007; 35: 429-438.

25. Petersen PE. Global policy for improvement of oral health in the 21 st century -implications to oral health research of World Health Assembly 2007, World Health Organization. Community Dent Oral Epidemiol 2009; 37: I-8.

26. Finlayson T, Siefert K, Ismail A, Sohn W. Psychosocial factors and early childhood caries among low - income African - American children in Detroit. Community Dent Oral Epidemiol 2007; 35: 439-448.

27. Cook L, Martinez-Mier E, Dean J,Weddell J, Sanders B, Eggertsson $\mathrm{H}$, et al. Dental caries experience and association to risk indicators of remote rural populations. International Journal of Paediatric Dentistry 2008; 18: 275-283. 